PROCEEDINGS OF THE

AMERICAN MATHEMATICAL SOCIETY

Volume 130, Number 4 , Pages 1079-1086

S 0002-9939(01)06319-5

Article electronically published on September 14, 2001

\title{
TIME DELAYED PARABOLIC SYSTEMS WITH COUPLED NONLINEAR BOUNDARY CONDITIONS
}

\author{
C. V. PAO
}

(Communicated by David S. Tartakoff)

\begin{abstract}
The aim of this paper is to show the existence and uniqueness of a solution for a system of time-delayed parabolic equations with coupled nonlinear boundary conditions. The time delays are of discrete type which may appear in the reaction function as well as in the boundary function. The approach to the problem is by the method of upper and lower solutions for nonquasimonotone functions.
\end{abstract}

\section{InTRODUCTION}

Parabolic partial differential equations with time delays have been given considerable attention in recent years, and various methods have been used for studying the existence and stability of the problem (cf. 11,2, 4, 5, 6, 7, 8, 9, 10, 11, 12, 13, 14, 15). Most of the earlier works are devoted to scalar parabolic equations using semi-group theory and functional analytic approach (cf. [2, 7, 12, 14, 15]). The work in 7 treats a coupled system of functional parabolic equations using the theory of dynamic flow for quasimonotone nondecreasing functions. Recently, the method of upper and lower solutions has been used to treat a class of time-delayed parabolic equations for mixed quasimonotone functions, including quasimonotone nondecreasing reaction functions (cf. 4, 9, 10). In all the above works the boundary condition is linear and the time delays appear only in the reaction function. In this paper we consider a system of time-delayed parabolic equations with coupled nonlinear boundary conditions where the reaction function and boundary function are not necessarily quasimonotone and the time delays may appear in the reaction function as well as in the boundary function. Our approach to the problem is by the method of upper and lower solutions for nonquasimonotone functions.

To describe the system we consider a bounded domain $\Omega$ in $\mathbb{R}^{n}$ with boundary $\partial \Omega$ and some positive constant vectors $\tau \equiv\left(\tau_{1}, \cdots, \tau_{N}\right), \tau^{\prime} \equiv\left(\tau_{1}^{\prime}, \cdots, \tau_{N}^{\prime}\right)$, representing the time delays. For any finite $T>0$, we set

$$
\begin{aligned}
& D_{T}=(0, T], \quad S_{T}=(0, T] \times \partial \Omega, \quad \bar{D}_{T}=[0, T] \times \bar{\Omega}, \\
& Q_{0}^{(i)}=\left[-\bar{\tau}_{i}, 0\right] \times \Omega, \quad \bar{Q}_{T}^{(i)}=\left[-\bar{\tau}_{i}, T\right] \times \bar{\Omega}, \quad \bar{Q}_{T}=\bar{Q}_{T}^{(1)} \times \cdots \times \bar{Q}_{T}^{(N)},
\end{aligned}
$$

Received by the editors October 5, 2000

1991 Mathematics Subject Classification. Primary 35K50; Secondary 35K57.

Key words and phrases. Parabolic systems, time delays, nonlinear boundary conditions, existence-uniqueness, nonquasimonotone functions, upper and lower solutions. 
where $\bar{\Omega}=\Omega \cup \partial \Omega$ and $\bar{\tau}_{i}=\max \left\{\tau_{i}, \tau_{i}^{\prime}\right\}, i=1, \cdots, N$. Denote by $C\left(\bar{D}_{T}\right)$ the set of continuous functions on $\bar{D}_{T}$ and let $\mathcal{C}\left(\bar{D}_{T}\right)=C\left(\bar{D}_{T}\right) \times \cdots \times C\left(\bar{D}_{T}\right)$ taken $N$-times. For any $\mathbf{u} \equiv\left(u_{1}, \cdots, u_{N}\right)$ in $\mathcal{C}\left(\bar{D}_{T}\right)$ we write

$$
\begin{aligned}
& \mathbf{u}_{\tau} \equiv \mathbf{u}_{\tau}(t, x) \equiv\left(u_{1}\left(t-\tau_{1}, x\right), \cdots, u_{N}\left(t-\tau_{N}, x\right)\right), \\
& \mathbf{u}_{\tau^{\prime}} \equiv \mathbf{u}_{\tau^{\prime}}(t, x) \equiv\left(u_{1}\left(t-\tau_{1}^{\prime}, x\right), \cdots, u_{N}\left(t-\tau_{N}^{\prime}, x\right)\right) .
\end{aligned}
$$

Then the system of time-delayed parabolic equations is given in the form

$$
\begin{array}{ll}
\partial u_{i} / \partial t-L_{i} u_{i}=f_{i}\left(t, x, \mathbf{u}, \mathbf{u}_{\tau}\right) & \text { in } D_{T}, \\
B_{i} u_{i}=g_{i}\left(t, x, \mathbf{u}, \mathbf{u}_{\tau^{\prime}}\right) & \text { on } S_{T}, \\
u_{i}(t, x)=\eta_{i}(t, x) & \text { in } Q_{0}^{(i)}(i=1, \cdots, N),
\end{array}
$$

where for each $i, L_{i}$ and $B_{i}$ are the (uniform) elliptic and boundary operators given by

$$
\begin{aligned}
L_{i} u_{i} & =\sum_{j, k=1}^{n} a_{j, k}^{(i)}(t, x) \frac{\partial^{2} u_{i}}{\partial x_{j} \partial x_{k}}+\sum_{j=1}^{n} b_{j}^{(i)}(t, x) \frac{\partial u_{i}}{\partial x_{j}}+c_{i}(t, x) u_{i}, \\
B_{i} u_{i} & \equiv \partial u_{i} / \partial \nu+\beta_{i}^{*} u_{i},
\end{aligned}
$$

with $\partial / \partial \nu$ denoting the outward normal derivative on $\partial \Omega$. It is assumed that $\partial \Omega$ is of class $C^{1+\alpha}$, and for each $i$ the coefficients of $L_{i}$ and the first partial derivatives of $a_{j, k}^{(i)}$ are Hölder continuous on $\bar{D}_{T}, c_{i} \leq 0$ and $\beta_{i}^{*} \geq 0$, and $\eta_{i}$ is Hölder continuous in $Q_{o}^{(i)}$. The functions $f_{i}(t, x, \cdot), g_{i}(t, x, \cdot)$ and $\beta_{i}^{*}(t, x)$ are Hölder continuous in their respective domains, and $f_{i}(\cdot, \mathbf{u}, \mathbf{v})$ and $g_{i}(\cdot, \mathbf{u}, \mathbf{v})$ satisfy the local Lipschitz condition (2.5) in Section 2. It is allowed that $L_{i}=0$ (and no boundary condition for the corresponding $u_{i}$ ) for some or all $i$. This means that problem (1.1) may consist of a combination of ordinary and parabolic equations.

The purpose of this paper is to show the existence and uniqueness of a solution to (1.1) by the method of upper and lower solutions. This existence result is obtained without any quasimonotone condition on $f_{i}\left(\cdot, \mathbf{u}, \mathbf{u}_{\tau}\right)$ or on $g_{i}\left(\cdot, \mathbf{u}, \mathbf{u}_{\tau^{\prime}}\right)$. We also show that in the special case where $f_{i}$ and $g_{i}$ possess a quasimonotone nondecreasing property then there exist two sequences which converge monotonically from above and below, respectively, to the unique solution. These results are stated in Section 2 , and proofs of these results are given in Section 3.

\section{The MAIN RESUlts}

In addition to the general smoothness assumptions in the Introduction we need a pair of coupled upper and lower solutions which are defined in the following.

Definition 2.1. Two smooth functions $\tilde{\mathbf{u}}=\left(\tilde{u}_{1}, \cdots, \tilde{u}_{N}\right)$ and $\hat{\mathbf{u}}=\left(\hat{u}_{1}, \cdots, \hat{u}_{N}\right)$ are called coupled upper and lower solutions of (1.1) if $\tilde{\mathbf{u}} \geq \hat{\mathbf{u}}$ on $\bar{Q}_{T}$ and if for each $i=1, \cdots, N$,

$$
\begin{array}{lll}
\partial \tilde{u}_{i} / \partial t-L_{i} \tilde{u}_{i} \geq f_{i}(t, x, \mathbf{u}, \mathbf{v}) & \text { for }(\mathbf{u}, \mathbf{v}) \in \mathcal{S} \times \mathcal{S}_{1} & \text { with } u_{i}=\tilde{u}_{i} \\
\partial \tilde{u}_{i} \partial \nu \geq g_{i}(t, x, \mathbf{u}, \mathbf{v}) & \text { for }(\mathbf{u}, \mathbf{v}) \in \mathcal{S} \times \mathcal{S}_{2} & \text { with } u_{i}=\tilde{u}_{i} \\
\partial \hat{u}_{i} / \partial t-L_{i} \hat{u}_{i} \leq f_{i}(t, x, \mathbf{u}, \mathbf{v}) & \text { for }(\mathbf{u}, \mathbf{v}) \in \mathcal{S} \times \mathcal{S}_{1} & \text { with } u_{i}=\hat{u}_{i} \\
\partial \hat{u}_{i} / \partial \nu \leq g_{i}(t, x, \mathbf{u}, \mathbf{v}) & \text { for }(\mathbf{u}, \mathbf{v}) \in \mathcal{S} \times \mathcal{S}_{2} & \text { with } u_{i}=\hat{u}_{i} \\
\tilde{u}_{i}(t, x) \geq \eta_{i}(t, x) \geq \hat{u}_{i}(t, x) & \text { in } Q_{o}^{(i)}
\end{array}
$$


In the above definition inequality between vectors is in the component-wise sense, and the smoothness of $\tilde{\mathbf{u}}, \hat{\mathbf{u}}$ is in the sense that these functions are continuously differentiable to the order appearing in (1.1). The subsets $\mathcal{S}, \mathcal{S}_{1}$ and $\mathcal{S}_{2}$ are given by

$$
\begin{aligned}
& \mathcal{S} \equiv\left\{\mathbf{u} \in \mathcal{C}\left(\bar{Q}_{T}\right) ; \hat{\mathbf{u}} \leq \mathbf{u} \leq \tilde{\mathbf{u}} \text { on } \bar{Q}_{T}\right\} \\
& \mathcal{S}_{1} \equiv\left\{\mathbf{v} \in \mathcal{C}\left(\bar{Q}_{T}\right) ; \hat{\mathbf{u}}_{\tau} \leq \mathbf{v}_{\tau} \leq \tilde{\mathbf{u}}_{\tau} \text { on } \bar{D}_{T}\right\} \\
& \mathcal{S}_{2} \equiv\left\{\mathbf{v} \in \mathcal{C}\left(\bar{Q}_{T}\right) ; \hat{\mathbf{u}}_{\tau^{\prime}} \leq \mathbf{v}_{\tau^{\prime}} \leq \tilde{\mathbf{u}}_{\tau^{\prime}} \text { on } \bar{D}_{T}\right\}
\end{aligned}
$$

Notice that if the $N$-vector functions

$$
\begin{aligned}
& \mathbf{f}(\cdot, \mathbf{u}, \mathbf{v}) \equiv\left(f_{1}(\cdot, \mathbf{u}, \mathbf{v}), \cdots, f_{N}(\cdot, \mathbf{u}, \mathbf{v})\right) \\
& \mathbf{g}(\cdot, \mathbf{u}, \mathbf{v}) \equiv\left(g_{1}(\cdot, \mathbf{u}, \mathbf{v}), \cdots, g_{N}(\cdot, \mathbf{u}, \mathbf{v})\right)
\end{aligned}
$$

are quasimonotone nondecreasing in $\mathcal{S} \times \mathcal{S}_{1}$ and $\mathcal{S} \times \mathcal{S}_{2}$ respectively (that is, for each $i=1, \cdots, N, f_{i}(\cdot, \mathbf{u}, \mathbf{v})$ and $g_{i}(\cdot, \mathbf{u}, \mathbf{v})$ are nondecreasing with respect to all the components of $\mathbf{u}$ and $\mathbf{v}$ except $\left.u_{i}\right)$, then the inequalities for $\tilde{\mathbf{u}}$ in (2.1) are reduced to

$$
\begin{array}{ll}
\partial \tilde{u}_{i} / \partial t-L_{i} \tilde{u}_{i} \geq f_{i}\left(t, x, \tilde{\mathbf{u}}, \tilde{\mathbf{u}}_{\tau}\right) & \text { in } D_{T}, \\
\partial \tilde{u}_{i} / \partial \nu \geq g_{i}\left(t, x, \tilde{\mathbf{u}}, \tilde{\mathbf{u}}_{\tau^{\prime}}\right) & \text { on } S_{T}, \\
\tilde{u}_{i}(t, x) \geq \eta_{i}(t, x) & \text { in } Q_{o}^{(i)} \quad(i=1, \cdots, N),
\end{array}
$$

and those for $\hat{\mathbf{u}}$ are reduced to (2.4) with all the inequalities reversed. Similar inequalities can be obtained if $\mathbf{f}(\cdot, \mathbf{u}, \mathbf{v})$ and $\mathbf{g}(\cdot, \mathbf{u}, \mathbf{v})$ are mixed quasimonotone functions (e.g., see [9, 10]). We assume that a pair of coupled upper and lower solutions $\tilde{\mathbf{u}}, \hat{\mathbf{u}}$ exist, and for each $i=1, \cdots, N$ there exist positive constants $K_{i}, K_{i}^{\prime}$ such that

$$
\begin{aligned}
\left|f_{i}(t, x, \mathbf{u}, \mathbf{v})-f_{i}\left(t, x, \mathbf{u}^{\prime}, \mathbf{v}^{\prime}\right)\right| \leq & K_{i}\left(\left|\mathbf{u}-\mathbf{u}^{\prime}\right|+\left|\mathbf{v}-\mathbf{v}^{\prime}\right|\right) \\
& \text { for }(\mathbf{u}, \mathbf{v}),\left(\mathbf{u}^{\prime}, \mathbf{v}^{\prime}\right) \in \mathcal{S} \times \mathcal{S}_{1}, \\
\left|g_{i}(t, x, \mathbf{u}, \mathbf{v})-g_{i}\left(t, x, \mathbf{u}^{\prime}, \mathbf{v}^{\prime}\right)\right| \leq & K_{i}^{\prime}\left(\left|\mathbf{u}-\mathbf{u}^{\prime}\right|+\left|\mathbf{v}-\mathbf{v}^{\prime}\right|\right) \\
& \text { for }(\mathbf{u}, \mathbf{v}),\left(\mathbf{u}^{\prime}, \mathbf{v}^{\prime}\right) \in \mathcal{S} \times \mathcal{S}_{2},
\end{aligned}
$$

where $|\mathbf{w}|=\left|w_{1}\right|+\cdots+\left|w_{N}\right|$ for any $\mathbf{w}=\left(w_{1}, \cdots, w_{N}\right)$ in $\mathbb{R}^{N}$. Our main existenceuniqueness result is the following.

Theorem 2.1. Let $\tilde{\mathbf{u}}, \hat{\mathbf{u}}$ be a pair of coupled upper and lower solutions of (1.1), and let condition (2.5) hold. Then there exists a unique solution $\mathbf{u}^{*} \equiv\left(u_{1}^{*}, \cdots, u_{N}^{*}\right) \in \mathcal{S}$ to $(1.1)$.

If the functions $\mathbf{f}(\cdot, \mathbf{u}, \mathbf{v}), \mathbf{g}(\cdot, \mathbf{u}, \mathbf{v})$ are quasimonotone nondecreasing, then for any initial iteration $\mathbf{u}^{(0)}$ we can construct a sequence $\left\{\mathbf{u}^{(k)}\right\} \equiv\left\{u_{1}^{(k)}, \cdots, u_{N}^{(k)}\right\}$ from the linear iteration process

$$
\begin{array}{ll}
\mathcal{L}_{i} u_{i}^{(k)}=F_{i}\left(t, x, \mathbf{u}^{(k-1)}, \mathbf{u}_{\tau}^{(k-1)}\right) & \text { in } D_{T}, \\
\mathcal{B}_{i} u_{i}^{(k)}=G_{i}\left(t, x, \mathbf{u}^{(k-1)}, \mathbf{u}_{\tau^{\prime}}^{(k-1)}\right) & \text { on } S_{T}, \\
u_{i}^{(k)}(t, x)=\eta_{i}(t, x) & \text { in } Q_{o}^{(i)} \quad(i=1, \cdots, N),
\end{array}
$$


where

(2.7)

$$
\begin{array}{r}
\mathcal{L}_{i} u_{i}=\partial u_{i} / \partial t-L_{i} u_{i}+K_{i} u_{i}, \quad \mathcal{B}_{i} u_{i}=B_{i} u_{i}+K_{i}^{\prime} u_{i}, \\
f_{i}\left(t, x, \mathbf{u}, \mathbf{u}_{\tau}\right)=K_{i} u_{i}+f_{i}\left(t, x, \mathbf{u}, \mathbf{u}_{\tau}\right), \quad G_{i}\left(t, x, \mathbf{u}, \mathbf{u}_{\tau^{\prime}}\right)=K_{i}^{\prime} u_{i}+g_{i}\left(t, x, \mathbf{u}, \mathbf{u}_{\tau^{\prime}}\right) \\
(i=1, \cdots, N)
\end{array}
$$

and $K_{i}$ and $K_{i}^{\prime}$ are the Lipschitz constants in (2.5). Denote the sequence by $\left\{\overline{\mathbf{u}}^{(k)}\right\}$ if $\mathbf{u}^{(o)}=\tilde{\mathbf{u}}$, and by $\left\{\underline{\mathbf{u}}^{(k)}\right\}$ if $\mathbf{u}^{(o)}=\hat{\mathbf{u}}$. Then we have the following monotone convergence of these sequences.

Theorem 2.2. Let the conditions in Theorem 2.1 be satisfied. If, in addition, $\mathbf{f}(\cdot, \mathbf{u}, \mathbf{v})$ and $\mathbf{g}(\cdot, \mathbf{u}, \mathbf{v})$ are quasimonotone nondecreasing in $\mathcal{S} \times \mathcal{S}_{1}$ and $\mathcal{S} \times \mathcal{S}_{2}$, respectively, then the sequences $\left\{\overline{\mathbf{u}}^{(k)}\right\},\left\{\underline{\mathbf{u}}^{(k)}\right\}$ converge monotonically to the unique solution $\mathbf{u}^{*} \in \mathcal{S}$. Moreover,

$$
\hat{\mathbf{u}} \leq \underline{\mathbf{u}}^{(k)} \leq \underline{\mathbf{u}}^{(k+1)} \leq \mathbf{u}^{*} \leq \overline{\mathbf{u}}^{(k+1)} \leq \overline{\mathbf{u}}^{(k)} \leq \tilde{\mathbf{u}} \quad \text { on } \bar{Q}_{T}
$$

for every $k=1,2, \cdots$.

Remark 2.1. Theorems 2.1 and 2.2 remain true if some of the boundary conditions in (1.1) are replaced by the Dirichlet condition $u_{i}=g_{i}(t, x)$. The proof for this case is the same as that given in Section 3 except with a slightly different integral representation from that in (3.5) (see [3, 9, 10]).

As an application of the above theorems we consider a two-compartment model in generic repression which is given by (cf. [6, 11, 15])

$$
\begin{aligned}
& \partial u_{1} / \partial t+\left(a_{1}+b_{1}\right) u_{1}=a_{1} u_{2}+f\left(v_{1}\left(t-\tau_{1}, x\right)\right), \\
& \partial v_{1} / \partial t+\left(a_{2}+b_{2}\right) v_{1}=a_{2} v_{2}, \\
& \partial u_{2} / \partial t-D_{1} \nabla^{2} u_{2}+b_{2} u_{2}=0, \\
& \partial v_{2} / \partial t-D_{2} \nabla^{2} v_{2}+b_{2} v_{2}=c_{o} u_{2}\left(t-\tau_{2}, x\right) \quad \text { in } D_{T}, \\
& \partial u_{2} / \partial \nu+\beta_{1} u_{2}=\beta_{1} u_{1}, \quad \partial v_{2} / \partial \nu+\beta_{2} v_{2}=\beta_{2} v_{1} \quad \text { on } S_{T}, \\
& u_{1}(0, x)=\eta_{1}(0, x), \quad v_{1}(t, x)=\eta_{1}^{*}(t, x), \quad\left(-\tau_{1} \leq t \leq 0\right), \\
& u_{2}(t, x)=\eta_{2}(t, x), \quad\left(-\tau_{2} \leq t \leq 0\right), \quad v_{2}(0, x)=\eta_{2}^{*}(0, x),
\end{aligned}
$$

where $a_{i}, b_{i}, \beta_{i}$ and $c_{o}, i=1,2$, are all positive constants. The function $f\left(v_{1}\right)$ is given by $f\left(v_{1}\right)=\sigma\left(1+c_{1} v_{1}^{\rho}\right)^{-1}$ with $\sigma$ and $c_{1}$ being the kinetic constants and $\rho \geq 1$ being the order of repression (cf. [6, [11]). It is easy to see by considering (2.9) with $\mathbf{u}=\left(u_{1}, v_{1}, u_{2}, v_{2}\right)$ and $L_{1}=L_{2}=0$ that for any positive constants $M, M^{*}$ satisfying

$$
M \geq \max \left\{\eta_{1}, \eta_{2}, \sigma / b_{1}\right\}, \quad M^{*} \geq\left\{\eta_{1}^{*}, \eta_{2}^{*}, c_{o} M / b_{2}\right\},
$$

the pair $\tilde{\mathbf{u}}=\left(M, M^{*}, M, M^{*}\right)$ and $\hat{\mathbf{u}}=(0,0,0,0)$ are coupled upper and lower solutions. Since the Lipschitz condition (2.5) is clearly satisfied by the functions at the right-hand side of (2.9), Theorem 2.1 ensures that for any non-negative function $\boldsymbol{\eta}(t, x)$, problem (2.9) has a unique bounded global solution $\mathbf{u}^{*} \equiv\left(u_{1}^{*}, v_{1}^{*}, u_{2}^{*}, v_{2}^{*}\right)$. A similar global existence result can be obtained for the three-compartment model in [6], including some improved upper and lower solutions which can be used to study the asymptotic behavior of the solution. 


\section{Proof of the theorems}

To prove Theorem 2.1 we first consider an equivalent system of (1.1) given by

$$
\begin{array}{ll}
\mathcal{L}_{i} u_{i}=F_{i}\left(t, x, \mathbf{u}, \mathbf{u}_{\tau}\right) & \text { in } D_{T}, \\
\mathcal{B}_{i} u_{i}=G_{i}\left(t, x, \mathbf{u}, \mathbf{u}_{\tau^{\prime}}\right) & \text { on } S_{T}, \\
u_{i}(t, x)=\eta_{i}(t, x) & \text { in } Q_{o}^{(i)}(i=1, \cdots, N),
\end{array}
$$

where $\mathcal{L}_{i}, \mathcal{B}_{i}, F_{i}$ and $G_{i}$ are given by (2.7). It is clear from (2.1) and (2.7) that

$$
\begin{array}{ll}
\mathcal{L}_{i} \tilde{u}_{i} \geq F_{i}(t, x, \mathbf{u}, \mathbf{v}) & \text { for }(\mathbf{u}, \mathbf{v}) \in \mathcal{S} \times \mathcal{S}_{1} \text { with } u_{i}=\tilde{u}_{i}, \\
\mathcal{B}_{i} \tilde{u}_{i} \geq G_{i}(t, x, \mathbf{u}, \mathbf{v}) & \text { for }(\mathbf{u}, \mathbf{v}) \in \mathcal{S} \times \mathcal{S}_{2} \text { with } u_{i}=\tilde{u}_{i},
\end{array}
$$

and a similar relation for $\hat{u}_{i}$. We show that problem (3.1) has a unique solution if $F_{i}(\cdot, \mathbf{u}, \mathbf{v})$ and $G_{i}(\cdot, \mathbf{u}, \mathbf{v})$ satisfy the following hypothesis.

Hypothesis $\mathbf{( H )}$. For each $i=1, \cdots, N, F_{i}(t, x, \mathbf{u}, \mathbf{v})$ and $G_{i}(t, x, \mathbf{u}, \mathbf{v})$ are uniformly bounded on $\bar{D}_{T} \times \mathbb{R}^{N} \times \mathbb{R}^{N}$ and satisfy the global Lipschitz condition

$$
\begin{aligned}
& \left|F_{i}(t, x, \mathbf{u}, \mathbf{v})-F_{i}\left(t, x, \mathbf{u}^{\prime}, \mathbf{v}^{\prime}\right)\right| \leq \bar{K}_{i}\left(\left|\mathbf{u}-\mathbf{u}^{\prime}\right|+\left|\mathbf{v}-\mathbf{v}^{\prime}\right|\right), \\
& \left|G_{i}(t, x, \mathbf{u}, \mathbf{v})-G_{i}\left(t, x, \mathbf{u}^{\prime}, \mathbf{v}^{\prime}\right)\right| \leq \bar{K}_{i}^{\prime}\left(\left|\mathbf{u}-\mathbf{u}^{\prime}\right|+\left|\mathbf{v}-\mathbf{v}^{\prime}\right|\right) \\
& \text { for all } \mathbf{u}, \mathbf{u}^{\prime}, \mathbf{v}, \mathbf{v}^{\prime} \text { in } \mathbb{R}^{N} .
\end{aligned}
$$

Let $\Gamma_{i}(t, x ; s, \xi)$ be the fundamental solution of $\mathcal{L}_{i}$, and let

$$
\begin{aligned}
& J_{i}^{(o)}(t, x) \equiv \int_{\Omega} \Gamma_{i}(t, x ; 0, \xi) \eta_{i}(0, \xi) d \xi \\
& \left(F_{i}\left(\mathbf{u}, \mathbf{u}_{\tau}\right)\right)(t, x) \equiv F_{i}\left(t, x, \mathbf{u}(t, x), \mathbf{u}_{\tau}(t, x)\right) \\
& \left(G_{i}\left(\mathbf{u}, \mathbf{u}_{\tau^{\prime}}\right)\right)(t, x) \equiv G_{i}\left(t, x, \mathbf{u}(t, x), \mathbf{u}_{\tau^{\prime}}(t, x)\right) \quad(i=1, \cdots, N),
\end{aligned}
$$

where $\mathbf{u}(t, x)=\boldsymbol{\eta}(t, x)$ when $t \leq 0$ (cf. [3, 8]). Define an operator $A_{i}: \mathcal{C}\left(\bar{D}_{T}\right) \rightarrow$ $C\left(\bar{D}_{T}\right)$ by

$$
\begin{aligned}
\left(A_{i} \mathbf{u}\right)(t, x)= & J_{i}^{(o)}(t, x)+\int_{o}^{t} d s \int_{\Omega} \Gamma_{i}(t, x ; s, \xi)\left(F_{i}\left(\mathbf{u}, \mathbf{u}_{\tau}\right)\right)(s, \xi) d \xi \\
& +\int_{o}^{t} d s \int_{\partial \Omega} \Gamma_{i}(t, x ; s, \xi) \psi_{i}(s, \xi) d \xi \quad(i=1, \cdots, N),
\end{aligned}
$$

where $\psi_{i}(t, x)$ is the density of the single layer potential and it is associated with the functions $F_{i}\left(\mathbf{u}, \mathbf{u}_{\tau}\right)$ and $G_{i}\left(\mathbf{u}, \mathbf{u}_{\tau^{\prime}}\right)$ (cf. [8], pg. 495). In the case $L_{i}=0, A_{i}$ is defined by

$$
\left(A_{i} \mathbf{u}\right)(t, x)=\eta_{i}(0, x)+\int_{0}^{t}\left(F\left(\mathbf{u}, \mathbf{u}_{\tau}\right)\right)(s, x) d s \quad(x \in \bar{\Omega}) .
$$

By the integral representation for parabolic boundary value problems a solution of (3.1), if it exists, may be expressed as

$$
u_{i}=A_{i} \mathbf{u} \quad \text { on } \bar{D}_{T} \quad(i=1, \cdots, N) .
$$

Define $\mathcal{A}: B_{r} \rightarrow \mathcal{C}\left(\bar{D}_{T}\right)$ by

$$
\mathcal{A} \mathbf{u}=\left(A_{1} \mathbf{u}, \cdots, A_{N} \mathbf{u}\right) \quad\left(\mathbf{u} \in B_{r}\right),
$$

where $B_{r}$ is the ball in $\mathcal{C}\left(\bar{D}_{T}\right)$ with radius $r>0$. Then equation (3.7) has a unique solution in $B_{r}$ if $\mathcal{A}$ has a unique fixed point in $B_{r}$. We show this in the following. 
Lemma 3.1. Under the hypothesis $(\mathrm{H})$ the integral system (3.7) has a unique solution $\mathbf{u}^{*}=\left(u_{1}^{*}, \cdots, u_{N}^{*}\right)$ in $B_{r}$ for some $r>0$. Moreover, $\mathbf{u}^{*}$ is the unique solution of $(3.1)$.

Proof. By (3.5) and the positive property of $\Gamma_{i}$,

$$
\begin{aligned}
\left|A_{i} \mathbf{u}\right| \leq\left|J_{i}^{(o)}\right| & +\int_{o}^{t} d s \int_{\Omega} \Gamma_{i}(t, x ; s, \xi)\left|F_{i}\left(\mathbf{u}, \mathbf{u}_{\tau}\right)\right| d \xi \\
& +\int_{o}^{t} d s \int_{\partial \Omega} \Gamma_{i}(t, x ; s, \xi)\left|\psi_{i}(s, \xi)\right| d \xi
\end{aligned}
$$

Since $\mathbf{u}_{\tau}$ and $\mathbf{u}_{\tau^{\prime}}$ are known when $t \leq 0$, Hypothesis $(\mathrm{H})$ implies that $F_{i}\left(\mathbf{u}, \mathbf{u}_{\tau}\right)$ and $G_{i}\left(\mathbf{u}, \mathbf{u}_{\tau^{\prime}}\right)$ are uniformly bounded for all $\mathbf{u}, \mathbf{u}_{\tau}$ and $\mathbf{u}_{\tau^{\prime}}$ in $\mathcal{C}\left(\bar{D}_{T}\right)$. By Lemma 9.6.1 of [8], $\psi_{i}(t, x)$ is bounded and continuous on $S_{T}$. In view of

$$
\int_{o}^{t} d s \int_{\Omega} \Gamma_{i}(t, x ; s, \xi) d \xi+\int_{o}^{t} d s \int_{\partial \Omega} \Gamma_{i}(t, x ; s, \xi) d \xi \leq C_{i}
$$

for some constant $C_{i}$, the uniform boundedness of $F_{i}\left(\mathbf{u}, \mathbf{u}_{\tau}\right)$ and $\psi_{i}(t, x)$ ensures the existence of $r_{i}>o$ such that $\left|A_{i} \mathbf{u}\right| \leq r_{i}$ for all $\mathbf{u} \in \mathcal{C}\left(\bar{D}_{T}\right)$. It is obvious from (3.6) that this relation also holds if $L_{i}=0$. By the definition of $\mathcal{A}$ we obtain $\|\mathcal{A} \mathbf{u}\| \leq r$, where $r=r_{1}+\cdots+r_{N}$ and $\|\cdot\|$ is the (maximum) norm in $\mathcal{C}\left(\bar{D}_{T}\right)$.

Let $\psi_{i}^{(l)}(t, x)$ be the density function corresponding to $F_{i}\left(\mathbf{u}^{(l)}, \mathbf{u}_{\tau}^{(l)}\right)$ and $G_{i}\left(\mathbf{u}^{(l)}, \mathbf{u}_{\tau^{\prime}}^{(l)}\right.$, where $l=1,2$. By Lemma 9.6.1 of [8] there exists a constant $K_{i}^{*}$ such that

$$
\left|\psi_{i}^{(1)}(t, x)-\psi_{i}^{(2)}(t, x)\right| \leq K_{i}^{*}\left\|\mathbf{u}^{(1)}-\mathbf{u}^{(2)}\right\|
$$

Using this relation and the argument in the proof of Lemma 9.6.2 in [8], we conclude that $\mathcal{A}$ is a compact operator on $B_{r}$ into itself. The existence of a fixed point $\mathbf{u}^{*} \in B_{r}$ follows from the Schauder fixed point theorem. Finally, by the argument in the proof of Theorem 9.6.1 in [8] $\mathbf{u}^{*}$ is a classical solution of (3.1).

To show the uniqueness of the solution we let $\tau_{o}=\min \left\{\tau_{1}, \cdots, \tau_{N}, \tau_{1}^{\prime}, \cdots, \tau_{N}^{\prime}\right\}$ and consider problem (3.7) in the domain $D_{1} \equiv\left(0, \tau_{o}\right] \times \Omega$ (that is, with $T=\tau_{o}$ ). Since $\mathbf{u}_{\tau}$ and $\mathbf{u}_{\tau^{\prime}}$ are known in $D_{1}$, the argument in the proof of Theorem 9.6.1 in [8] shows that the solution $\mathbf{u}^{*}$ is unique on $\bar{D}_{1} \equiv\left[0, r_{o}\right] \times \bar{\Omega}$. Knowing the uniqueness of the solution in $\bar{D}_{1}$, a ladder argument ensures that $\mathbf{u}^{*}$ is the unique solution of $(3.7)$ on $\bar{D}_{m}=\left(0, m \tau_{o}\right] \times \bar{\Omega}$ for every integer $m=1,2, \cdots$. This proves the lemma.

Proof of Theorem 2.1. The proof is based on Lemma 3.1 for some modified functions $\hat{F}_{i}\left(\cdot, \mathbf{u}, \mathbf{u}_{\tau}\right)$ and $\hat{G}_{i}\left(\cdot, \mathbf{u}, \mathbf{u}_{\tau^{\prime}}\right)$ which are defined to coincide with $F_{i}\left(\cdot, \mathbf{u}, \mathbf{u}_{\tau}\right)$ on $\mathcal{S} \times \mathcal{S}_{1}$ and with $G_{i}\left(\cdot, \mathbf{u}, \mathbf{u}_{\tau^{\prime}}\right)$ on $\mathcal{S} \times \mathcal{S}_{2}$ and satisfy the conditions in Hypothesis $(\mathrm{H})$. For example, if we denote by $[\mathbf{u}]_{\sigma}$ a vector in $\mathbb{R}^{\sigma}$ with $\sigma$ components of $\mathbf{u} \in \mathbb{R}^{N}$ and white $\mathbf{u}$ in the split form

$$
\mathbf{u}=\left([\mathbf{u}]_{a_{i}},[\mathbf{u}]_{b_{i}},[\mathbf{u}]_{c_{i}}\right) \quad(i=1, \cdots, N)
$$

for each $i$, where $a_{i}, b_{i}$, and $c_{i}$ are nonnegative integers satisfying $a_{i}+b_{i}+c_{i}=N$ and

$$
[\mathbf{u}]_{a_{i}}>[\tilde{\mathbf{u}}]_{a_{i}}, \quad[\tilde{\mathbf{u}}]_{b_{i}} \geq[\mathbf{u}]_{b_{i}} \geq[\hat{\mathbf{u}}]_{b_{i}}, \quad[\mathbf{u}]_{c_{i}}<[\hat{\mathbf{u}}]_{c_{i}}
$$


then a possible choice of $\hat{F}_{i}, \hat{G}_{i}$ are the truncated functions given by

$$
\begin{aligned}
& \hat{F}_{i}\left(\cdot, \mathbf{u}, \mathbf{u}_{\tau}\right) \equiv F_{i}\left([\tilde{\mathbf{u}}]_{a_{i}},[\mathbf{u}]_{b_{i}},[\hat{\mathbf{u}}]_{c_{i}},\left[\tilde{\mathbf{u}}_{\tau}\right]_{a_{i}},\left[\mathbf{u}_{\tau}\right]_{b_{i}},\left[\hat{\mathbf{u}}_{\tau}\right]_{c_{i}}\right), \\
& \hat{G}_{i}\left(\cdot, \mathbf{u}, \mathbf{u}_{\tau^{\prime}}\right) \equiv G_{i}\left([\tilde{\mathbf{u}}]_{a_{i}},[\mathbf{u}]_{b_{i}},[\hat{\mathbf{u}}]_{c_{i}},\left[\tilde{\mathbf{u}}_{\tau^{\prime}}\right]_{a_{i}},\left[\mathbf{u}_{\tau^{\prime}}\right]_{b_{i}},\left[\hat{\mathbf{u}}_{\tau^{\prime}}\right]_{c_{i}}\right) \\
& (i=1, \cdots, N),
\end{aligned}
$$

where $a_{i}, b_{i}$ and $c_{i}$ may be different for different $\mathbf{u}$. In other words, the modified functions $\hat{F}_{i}, \hat{G}_{i}$ are obtained from $F_{i}$ and $G_{i}$ by replacing the component $u_{j}$ by $\tilde{u}_{j}$ whenever $u_{j}>\tilde{u}_{j}$, and by $\hat{u}_{j}$ whenever $u_{j}<\hat{u}_{j}$. Hence the integer $a_{i}$ (resp., $c_{i}$ ) in the split form of $\mathbf{u}$ is the maximal number of components $u_{j}$ satisfying $u_{j}>\tilde{u}_{j}$ (resp., $u_{j}<\hat{u}_{j}$ ). The above definition implies that the truncated functions $\hat{F}_{i}$ and $\hat{G}_{i}$ satisfy the conditions in Hypothesis $(\mathrm{H})$. By Lemma 3.1 the modified problem of (3.1) (that is, problem (3.1) with $F_{i}, G_{i}$ replaced, respectively, by $\hat{F}_{i}$ and $\hat{G}_{i}$ ) has a unique solution $\mathbf{u}^{*}$. Hence to prove the theorem it suffices to show $\hat{\mathbf{u}} \leq \mathbf{u}^{*} \leq \tilde{\mathbf{u}}$ on $\bar{D}_{T}$.

Given any $\mathbf{u} \in \mathbb{R}^{N}$ we write $\mathbf{u}=\left(u_{i},[\mathbf{u}]_{N-1}\right)$. By the Lipschitz condition (2.5),

$$
\begin{aligned}
K_{i} \hat{u}_{i}+f_{i}\left(\cdot, \hat{u}_{i},[\mathbf{u}]_{N-1}, \mathbf{u}_{\tau}\right) & \leq K_{i} u_{i}+f_{i}\left(\cdot, u_{i},[\mathbf{u}]_{N-1}, \mathbf{u}_{\tau}\right) \\
& \leq K_{i} \tilde{u}_{i}+f\left(\cdot, \tilde{u}_{i},[\mathbf{u}]_{N-1}, \mathbf{u}_{\tau}\right) \quad \text { for }\left(\mathbf{u}, \mathbf{u}_{\tau}\right) \in \mathcal{S} \times \mathcal{S}_{1} .
\end{aligned}
$$

This implies that the truncated function $\hat{F}_{i}$ satisfies the relation

$$
\begin{array}{r}
\hat{F}_{i}\left(\hat{u}_{i},[\mathbf{u}]_{N-1}, \mathbf{u}_{\tau}\right) \leq \hat{F}_{i}\left(u_{i},[\mathbf{u}]_{N-1}, \mathbf{u}_{\tau}\right) \leq \hat{F}_{i}\left(\tilde{u}_{i},[\mathbf{u}]_{N-1}, \mathbf{u}_{\tau}\right) \\
\text { for all } \mathbf{u}, \mathbf{u}_{\tau} \in \mathbb{R}^{N} .
\end{array}
$$

A similar relation holds for $\hat{G}_{i}\left(u_{i},[\mathbf{u}]_{N-1}, \mathbf{u}_{\tau^{\prime}}\right)$. These relations and (3.2) yield

$$
\begin{array}{ll}
\mathcal{L}_{i} \tilde{u}_{i} \geq \hat{F}_{i}\left(\mathbf{u}, \mathbf{u}_{\tau}\right) & \text { in } D_{T}, \\
\mathcal{B}_{i} \tilde{u}_{i} \geq \hat{G}_{i}\left(\mathbf{u}, \mathbf{u}_{\tau^{\prime}}\right) & \text { on } S_{T}
\end{array}
$$

for all $\mathbf{u}, \mathbf{u}_{\tau}$ and $\mathbf{u}_{\tau^{\prime}}$ in $\mathbb{R}^{N}$. Similarly, $\hat{u}_{i}$ satisfies the inequalities in (3.11) in reversed order. Since $\mathbf{u}^{*}$ satisfies (3.1) when $F_{i}$ and $G_{i}$ are replaced by $\hat{F}_{i}$ and $\hat{G}_{i}$ we see from (3.11) that the function $w_{i} \equiv \tilde{u}_{i}-u_{i}^{*}$ satisfies the relation

$$
\begin{array}{ll}
\mathcal{L}_{i} w_{i}=\mathcal{L}_{i} \tilde{u}_{i}-\hat{F}_{i}\left(\mathbf{u}^{*}, \mathbf{u}_{\tau}^{*}\right) \geq 0 & \text { in } D_{T}, \\
\mathcal{B}_{i} w_{i}=\mathcal{B}_{i} \tilde{u}_{i}-\hat{G}_{i}\left(\mathbf{u}^{*}, \mathbf{u}_{\tau^{\prime}}^{*}\right) \geq 0 & \text { on } S_{T}, \\
w_{i}(0, x)=\tilde{u}_{i}(0, x)-\eta_{i}(0, x) \geq 0 & \text { in } \Omega \quad(i=1, \cdots, N) .
\end{array}
$$

By the positivity lemma for parabolic boundary value problems we obtain $w_{i} \geq 0$ on $\bar{D}_{T}, i=1, \cdots, N$. This proves $\mathbf{u}^{*} \leq \tilde{\mathbf{u}}$. A similar argument gives $\mathbf{u}^{*} \geq \hat{\mathbf{u}}$. This shows that $\mathbf{u}^{*}$ is the unique solution of the original problem (3.1). The equivalence between (3.1) and (1.1) ensures that $\mathbf{u}^{*}$ is the unique solution of (1.1) in $\mathcal{S}$. This completes the proof of the theorem.

Proof of Theorem 2.2. It is easily seen from the argument in 8] (see pg. 494) for parabolic boundary value problems without time delays that the sequences $\left\{\overline{\mathbf{u}}^{k}\right\},\left\{\underline{\mathbf{u}}^{k}\right\}$ goverened by (2.6) with $\overline{\mathbf{u}}^{(o)}=\tilde{\mathbf{u}}$ and $\underline{\mathbf{u}}^{(o)}=\hat{\mathbf{u}}$, respectively, possess the monotone property in (2.8). This implies that the pointwise limits

$$
\lim _{k \rightarrow \infty} \overline{\mathbf{u}}^{(k)}(t, x)=\overline{\mathbf{u}}(t, x), \quad \lim _{k \rightarrow \infty} \underline{\mathbf{u}}^{(k)}(t, x)=\underline{\mathbf{u}}(t, x)
$$


exist and satisfy relation (2.8) where $\mathbf{u}^{*}$ is replaced by $\overline{\mathbf{u}}$ or $\underline{\mathbf{u}}$. Since by (3.5)-(3.6) the sequence $\left\{\mathbf{u}^{(k)}\right\}$ given in (2.6) can be expressed as

$$
u_{i}^{(k)}=A_{i} \mathbf{u}^{(k-1)}, k=1,2, \cdots \quad(i=1, \cdots, N),
$$

the dominated convergence theorem ensures that $u_{i}=A_{i} \mathbf{u}, i=1, \cdots, N$, where $\mathbf{u}$ stands for either $\overline{\mathbf{u}}$ or $\underline{\mathbf{u}}$. A regularity argument as that in [8, 9] shows that $\overline{\mathbf{u}}$ and $\underline{\mathbf{u}}$ are solutions of (3.1). Finally, a ladder argument as that in the proof of Lemma 3.1 shows that $\overline{\mathbf{u}}=\underline{\mathbf{u}}$ and their common value is the unique solution of (1.1) in $\mathcal{S}$.

\section{REFERENCES}

1. J. M. Cushing, Integrodifferential Equations and Delay Models in Population Dynamics, Lecture Notes in Biomath., Vol. 20, Springer-Verlag, New York, 1977. MR 58:15300

2. W. E. Fitzgibbon, Semilinear functional differential equations in Banach space, J. Differential Equations, 29 (1978), 1-14. MR 58:11746

3. A. Friedman, Partial Differential Equations of Parabolic Type, Prentice-Hall, Englewood Cliffs, NJ, 1964. MR 31:6062

4. X. Lu, Persistence and extinction in a competition-diffusion system with time delays, Canadian Appl. Math. Quart., 2 (1994), 231-246. MR 95e:92016

5. S. Luckhaus, Global boundedness for a delay differential equation, Trans. Amer. Math. Soc., 294 (1986), 767-774. MR 87j:35053

6. J. M. Mahaffy and C. V. Pao, Models of genetic control by repression with time delays and spatial effects, J. Math. Biol., 20 (1984), 39-57. MR 85h:92011

7. R. H. Martin and H. L. Smith, Abstract functional differential equations and reaction diffusion systems, Trans. Amer. Math. Soc., 321 (1990), 1-44. MR 90m:35194]

8. C. V. Pao, Nonlinear Parabolic and Elliptic Equations, Plenum, New York, 1992. MR 94c:35002

9. C. V. Pao, Coupled nonlinear parabolic systems with time delays, J. Math. Anal. Appl., 196 (1995), 237-265. MR 96j:35260

10. C. V. Pao, Systems of parabolic equations with continuous and discrete delays, J. Math. Anal. Appl., 205 (1997), 157-185. MR 97j:35155

11. C. V. Pao, On a coupled reaction diffusion system with time delays, SIAM J. Math. Anal., 18 (1987), 1026-1039. MR 88d:35105

12. S. M. Rankin, Existence and asymptotic behavior of a functional differential equation in Banach space, J. Math. Anal. Appl., 88 (1982), 531-542. MR 84h:35160

13. R. Redlinger, Existence theorems for semilinear parabolic systems with functionals, Nonlinear Analysis, TMA, 8 (1984), 667-682. MR 86a:35080

14. C. C. Travis and G. F. Webb, Existence and stability for partial functional differential equations, Trans. Amer. Math. Soc., 200 (1974), 395-418. MR 52:3690

15. J. Wu, Theory and Applications of Partial Functional Differential Equations, Springer-Verlag, New York, 1996. MR 98a:35135

Department of Mathematics, North Carolina State University, Raleigh, North CarOLINA 27695-8205

E-mail address: cvpao@math.ncsu.edu 